\title{
Neuroprotective Role of Akt in Hypoxia Adaptation in Andeans
}

\author{
Helen Zhao', Jonathan Lin ${ }^{2,3,4}$, Gary Sieck ${ }^{5}$ and Gabriel G. Haddad ${ }^{1,6,7 *}$ \\ ${ }^{1}$ Department of Pediatrics (Respiratory Medicine), University of California, San Diego, La Jolla, CA, United States, \\ ${ }^{2}$ Department of Pathology, University of California, San Diego, La Jolla, CA, United States, ${ }^{3}$ Department of Pathology, \\ Stanford University, Stanford, CA, United States, ${ }^{4}$ VA Palo Alto Healthcare System, Palo Alto, CA, United States, \\ ${ }^{5}$ Department of Physiology and Biomedical Engineering, Mayo Clinic, Rochester, MN, United States, ${ }^{6}$ Department \\ of Neurosciences, University of California, San Diego, La Jolla, CA, United States, ${ }^{7}$ The Rady Children's Hospital, San \\ Diego, CA, United States
}

Chronic mountain sickness (CMS) is a disease that potentially threatens a large segment of high-altitude populations during extended living at altitudes above 2,500 m. Patients with CMS suffer from severe hypoxemia, excessive erythrocytosis and neurologic deficits. The cellular mechanisms underlying CMS neuropathology remain unknown. We previously showed that iPSC-derived CMS neurons have altered mitochondrial dynamics and increased susceptibility to hypoxia-induced cell death. Genome analysis from the same population identified many ER stress-related genes that play an important role in hypoxia adaptation or lack thereof. In the current study, we showed that iPSCderived CMS neurons have increased expression of ER stress markers Grp78 and XBP1s under normoxia and hyperphosphorylation of PERK under hypoxia, alleviating ER stress does not rescue the hypoxia-induced CMS neuronal cell death. Akt is a cytosolic regulator of ER stress with PERK as a direct target of Akt. CMS neurons exhibited lack of Akt activation and lack of increased Parkin expression as compared to nonCMS neurons under hypoxia. By enhancing Akt activation and Parkin overexpression, hypoxia-induced CMS neuronal cell death was reduced. Taken together, we propose that increased Akt activation protects non-CMS from hypoxia-induced cell death. In contrast, impaired adaptive mechanisms including failure to activate Akt and increase Parkin expression render CMS neurons more susceptible to hypoxia-induced cell death.

Keywords: chronic mountain sickness, iPSCs, neurons, Akt, Parkin, hypoxia, cell death

\section{INTRODUCTION}

Chronic Mountain Sickness (CMS) is a maladaptation to hypoxia that is characterized by severe hypoxemia, excessive erythrocytosis and many neurologic manifestations, including migraine, headache, mental fatigue, confusion, and memory loss. CMS disease threatens a large segment of the high-altitude population (more than 100 million highlanders) during extended living at altitudes above 2,500 $\mathrm{m}$. Since hypoxia is a common feature of many diseases at sea level including cardiovascular diseases, neurodegenerative diseases, stroke, diabetes and cancer, we believe that investigating the underlying mechanisms of hypoxia maladaptation or adaptation in CMS and non-CMS subjects may lead us to a better understanding of many hypoxia-related diseases as well. However, due to sample availability and ethical issues, our understanding of CMS neuropathology 
in humans remains largely unexplored at cellular and molecular levels. By using CMS disease-specific iPSC-derived neurons, we have previously reported mitochondrial dysfunction in CMS neurons under normoxia and an increased vulnerability to hypoxia-induced cell death after 48 h (Zhao et al., 2018). Here, we are interested in investigating the underlying mechanisms that contribute to hypoxia-induced cell death in CMS neurons.

Recent clinical studies have shown that CMS patients have blunted cerebral blood flow and exaggerated systemic oxidativeinflammatory-nitrosative stress with impaired cognition (Bao et al., 2017; Bailey et al., 2019) and CMS is believed to have clinical manifestations of aging at high altitude (Sime et al., 1975). In the brain, reduced cerebral blood flow and hypoxia disturb normal physiological functions and lead to metabolic stress. In conditions of stress including hypoxia, organisms activate endogenous adaptive cytoprotection mechanisms including the endoplasmic reticulum (ER) unfolded protein response (ER stress response), ER associated degradation (ERAD), autophagy, mitophagy, and/or mitochondrial biogenesis to restore cellular homeostasis (Senft and Ronai, 2015; Wu and Chen, 2015; Xu et al., 2015; Maekawa and Inagi, 2017; Delbrel et al., 2018; Yamashita and Kanki, 2018). Indeed, under pathological conditions, when an element of the endogenous adaptive cytoprotection mechanisms is impaired, the overall cellular homeostasis can be disturbed (Senft and Ronai, 2015).

Considering that the ER and mitochondria form a direct physical contact via mitochondria associated membrane (MAM) and functionally interact with each other (Bouman et al., 2011; Senft and Ronai, 2015), we hypothesize that mitochondrial dysfunction might therefore affect ER function as well as other endogenous adaptive mechanisms such as mitophagy. Akt is a pro-survival signaling protein and the PI3K/Akt/mTORC pathway cross links with both ER stress and autophagy (Qin et al., 2010; Kabir et al., 2018). In CMS patients, the Akt gene is shown to associate with chronic mountain sickness (Buroker et al., 2017) and plays an important role in regulating apoptosis in CMS patients with excessive erythrocytosis ( $\mathrm{Li}$ et al., 2017; Zhao et al., 2017a). Therefore, in the current study, we explored the relationship between mitochondria and ER stress in iPSCs-derived CMS neurons and nonCMS neurons and investigated the potential rescue role of Akt from progression to hypoxia-induced cell death in CMS neurons.

\section{MATERIALS AND METHODS}

\section{Ethics Statement}

All non-CMS $(n=3)$ and CMS $(n=3)$ subjects in the current study were adult males residing in the Andean mountain range, in Cerro de Pasco, Peru, at an elevation of more than 4,300 m. Each subject signed an informed written consent under protocols approved by the University of California San Diego and the Universidad Peruana Cayetano Heredia, and all experiments were performed in accordance with the relevant guidelines and regulations.

\section{iPSCs-Derived Neuronal Culture}

Detailed experimental procedures were previously described (Zhao et al., 2015). In brief, fibroblast cells obtained from individual's skin biopsies were reprogrammed into iPSCs using retrovirus containing the Yamanaka factors (OCT4, SOX2, KLF4, and $c$-MYC human cDNAs) (Takahashi et al., 2007). iPSCsderived neural progenitor cells (NPCs) were generated following an embryoid bodies (EBs) spin protocol (Kim et al., 2011b) with an AggreWell plate (Stem Cell Technologies, Canada) following the manufacturer's instructions. Rosette-bearing EBs were manually removed and dissociated into individual cells, which were then plated into a poly-L-ornithine/laminin coated plate to generate a monolayer of NPC culture. NPCs were expanded in DMEM/F12 medium containing $1 \times$ glutamax, $0.5 \times \mathrm{N} 2$, $0.5 \times$ B27, $1 \%$ penicillin/streptomycin, and $20 \mathrm{ng} / \mathrm{ml} \mathrm{FGF2.}$ Neural terminal differentiation was initiated by withdrawing FGF2 from DMEM/F12 medium when cells reach to $70 \%$ confluence. Most of the iPSCs-derived neurons using the current protocol were glutamatergic (>90\%) neurons (Zhao et al., 2015).

\section{Hypoxia Treatment}

All cultures were maintained in an incubator supplied with $21 \%$ $\mathrm{O}_{2}$ and $5 \% \mathrm{CO}_{2}$ at $37^{\circ} \mathrm{C}$. Three-week-old neurons were exposed to hypoxia $\left(1 \% \mathrm{O}_{2}\right)$ for 6,24 , or $48 \mathrm{~h}$.

\section{Chemical Treatment}

iPSCs-derived neurons were treated with an ER stress inducer either $1 \mu \mathrm{M}$ thapsigargin (TG, Sigma-Aldrich, \#T9033) or $5 \mu \mathrm{g} / \mathrm{ml}$ tunicamycin (TM, Sigma-Aldrich, \#T7765) for $24 \mathrm{~h}$ as previously described (Oslowski and Urano, 2011). $100 \mathrm{nM}$ Insulin (Roche, \#11376497001) was added $30 \mathrm{~min}$ before the hypoxia treatment and $1 \mathrm{mM}$ of 4-Phenylbutyric acid (4PBA, Sigma-Aldrich, \#P21005) was added at the beginning of hypoxia exposure (Liu et al., 2016). 5 MM MK2206 (Cayman $\# 1032350$-13-2) was added together with hypoxia treatment (Lin et al., 2018).

\section{Western Blot}

Neurons were homogenized with a glass-teflon homogenizer (Thomas Scientific) in RIPA buffer (Cell signaling \#9806S), plus protease and phosphatase inhibitors including $1 \mathrm{mM}$ phenylmethylsulfonyl fluoride (Sigma-Aldrich \# P7626) and $1 \times$ protease inhibitor cocktail (Thermo Scientific \#1861284). Homogenates were then centrifuged for $10 \mathrm{~min}$ at $10,000 \times g$ and $4^{\circ} \mathrm{C}$. The supernatants were collected and protein concentration was determined using a Bio-Rad protein assay kit (Bio-Rad). Twenty micrograms of protein were separated on NuPAGE ${ }^{\mathrm{TM}}{ }_{4-}$ $12 \%$ Bis-Tris Mini Protein Gel (Invitrogen \#NP0321) and then transferred to polyvinylidene difluoride membranes (Millipore \#IPVH00010). The membranes were probed with primary antibody in PBST with 5\% BSA overnight at $4^{\circ} \mathrm{C}$ and followed by an appropriate horseradish peroxidase-conjugated secondary antibody (Thermofisher Scientific \#A16160 and \#65-6120). Immunoreactive bands were visualized using Bio-Rad ChemiDoc XRS with enhanced chemiluminescence (Perkin-Elmer). Equal loading was assessed using GAPDH and data were analyzed using 
ImageLab software (version 3.0, Bio-Rad). TM- and TG- treated cells act as positive control for ER stress. CCCP-treated PC3 cell lysates (Cell signaling, \#21107s) was used as positive control for the western blot of PINK1 and phospho-Ubiquitin (Ser65).

The primary antibodies PERK (1:100, \#sc-377400), Grp78 (1:100, \#sc-376768), Akt (1:200, \#sc-5298), Parkin (1:200, \#sc32282), and Mfn2 (1:200, \#sc-100560) were purchased from Santa Cruz Biotechnology. Phospho-PERK (pPERK, 1:250, \#649401) and XBP1s (1:500, \#619502) were purchased from Biolegend. Phospho-Akt (pAkt, 1:1000, \#4060), cleaved PARP (1:1000, \#9541), CHOP (1:1000, \#2895), PINK1 (1:1000, \#6946) and GAPDH (1:1000, \#2118) were purchased from Cell Signaling. Phospho-Ubiquitin (Ser65) (pS65-Ub, 1:1000, \#ABS1513-l) was obtained from Millipore.

\section{Lentivirus Transduction}

The pLV [Exp]-EGFP: T2A: Puro-EF1A > hPRKN (NM_004562.3) construct was commercially generated by Vector Builder. The transduction was performed on NPCs and successfully transduced NPCs were selected with $1 \mu \mathrm{g} / \mathrm{ml}$ of puromycin (Clontech \#631306). Parkin overexpression was confirmed by western blot.

\section{Statistics}

Data were collected from three individuals in each group and from three experiments were performed in each individual. Results were expressed as group mean \pm SD. Differences in means were considered statistically significant when $p<0.05$ by independent $t$ tests or one-way ANOVA followed by HolmBonferroni post hoc analysis as appropriate.

\section{RESULTS}

\section{Increased ER Stress in CMS Neurons}

We have previously reported that CMS neurons had altered mitochondrial dynamics and function (Zhao et al., 2018). Due to the fact that ER and mitochondria form physical contact through MAM and interact with each other functionally (Bouman et al., 2011; Senft and Ronai, 2015), we examined whether the altered mitochondrial function activates the ER stress response. We first measured the expression of Grp78, a well-known marker for ER stress. Grp78 was indeed significantly increased in CMS neurons under normoxia and no difference was detected between non-CMS and CMS neurons under hypoxia (Figures 1A,B, $\left.{ }^{* *} p<0.01\right)$. Under stress conditions, accumulation of unfolded protein dissociates Grp 78 from ER stress sensor proteins, which activates ER stress signaling pathways including IRE1, activating transcription factor-6 (ATF6) and PERK. Both IRE1 and PERK pathways are widely explored and play a critical role in cell death (Szegezdi et al., 2006; Iurlaro et al., 2017). We therefore focused on these two pathways and compared the expression of $\mathrm{XBP} 1 \mathrm{~s}$, marker of inositol-requiring protein-1 (IRE1) pathway, and pPERK/PERK, markers of protein kinase RNA (PKR)-like ER kinase (PERK) pathway, in both CMS and non-CMS groups. A significantly higher XBP1s expression was observed in CMS neurons (Figures 1C,D, ${ }^{*} p<0.05$ ), indicating an increased IRE1mediated ER stress response in CMS neurons under normoxia.
The level of PERK phosphorylation (pPERK/PERK) is not different between non-CMS and CMS neurons under normoxia. However, a significantly higher extent of phosphorylation PERK was observed in CMS neurons after hypoxia treatment for $24 \mathrm{~h}$ (Figures 1E,F, \#\#p<0.01) and the difference between non-CMS and CMS was significant (Figures1 E,F, ${ }^{* *} p<0.01$ ), suggesting a hyperactivation of the PERK pathway of the ER stress response in CMS neurons under hypoxia.

To examine whether hypoxia-induced hyperactivation of the PERK pathway eventually results in an increased cell death of CMS neurons, we treated CMS neurons with the ER stress alleviator 4-Phenylbutyric acid (4-PBA) and exposed them to hypoxia for $48 \mathrm{~h}$, and then measured the expression of cleaved PARP (cPARP), an apoptosis marker (Koh et al., 2005). As shown in Figures 1G,H, 4-PBA treatment indeed inhibited phosphorylation of PERK $(\sim 61 \%$ decrease, $p<0.05)$ and 4 PBA treatment has a tendency to rescue hypoxia-induced cell death, but the $p$ value did not reach statistical significance (Figure 1H, $p=0.08$ ).

\section{Hypoxia Induces Akt Activation in Non-CMS Neurons}

Since (a) PERK is a direct Akt target and was hyperactivated in CMS neurons after hypoxia treatment (Blaustein et al., 2013), and (b) Akt has been shown to be associated with chronic mountain sickness (Buroker et al., 2017), we determined the level of Akt activation (pAkt S473/Akt) in both non-CMS and CMS neurons. As shown in Figures 2A,B, there was no difference between non-CMS neurons and CMS neurons in Akt activation $(p>0.05)$ at baseline. Hypoxia treatment induced Akt activation in non-CMS neurons significantly (Figures 2A,B, \#\# < 0.01) but did not in CMS neurons. Interestingly, the difference of Akt activation between non-CMS and CMS was significant at $24 \mathrm{~h}$ (Figures 2A,B and ${ }^{* *} p<0.01$ ).

\section{Differences in Parkin Expression Between CMS and Non-CMS Neurons Under Hypoxia}

Inhibition of Akt has been shown to affect Pink accumulation, and ubiquitination and Parkin recruitment in SHSY5Y cells and iPSC-derived neurons (Mccoy et al., 2014; Soutar et al., 2018). Both PINK1 and Parkin are important for mitochondrial quality control and mitophagy. Since the level of Akt activation in CMS neurons is significantly lower than that in non-CMS neurons under hypoxia, we then determined whether a lack of Akt activation in CMS affects PINK1 and Parkin expression. We first measured the expression of PINK1 and PINK1 substrate pS65-ubiquitin. Neither PINK1 nor PINK1 substrate pS65ubiquitin expression were detected in non-CMS or CMS neurons (Supplementary Figure 1), suggesting the lack of PINK1mediated ubiquitination in these neurons. Furthermore, we observed Parkin expression in both non-CMS and CMS neurons. As shown in Figures 2C,D, there was no difference in Parkin expression between non-CMS neurons and CMS neurons at baseline. Hypoxia alone did not change Parkin expression level in either non-CMS or CMS neurons (Figures 2C,D, $p>0.05$ ). Of note, however, there was a difference in Parkin expression 
A

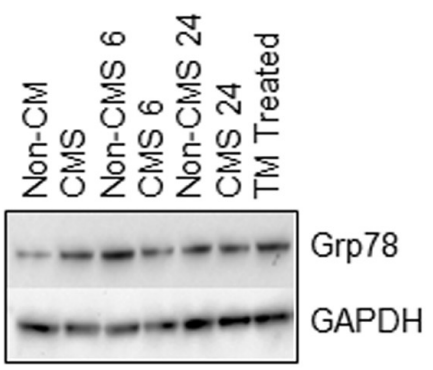

C

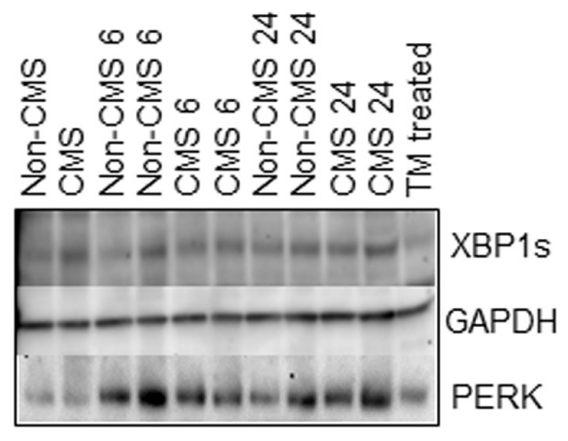

E

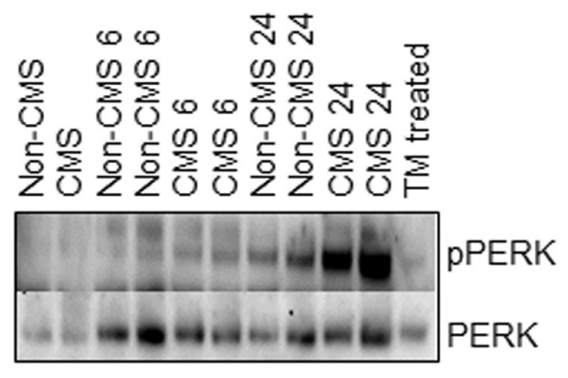

G

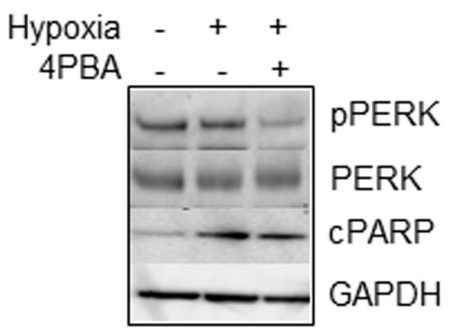

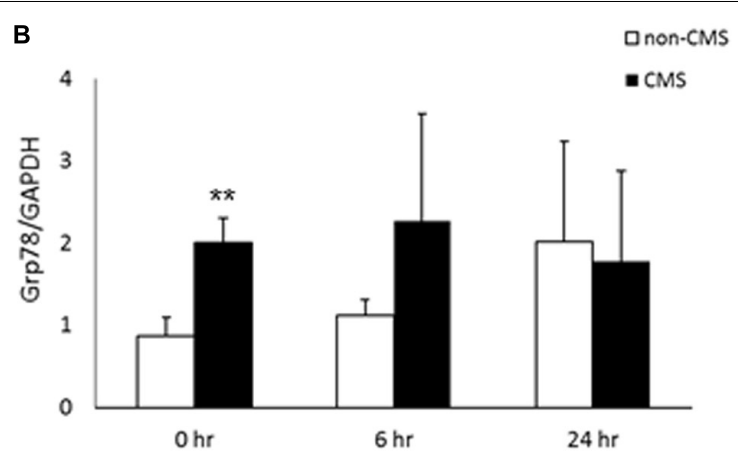

D
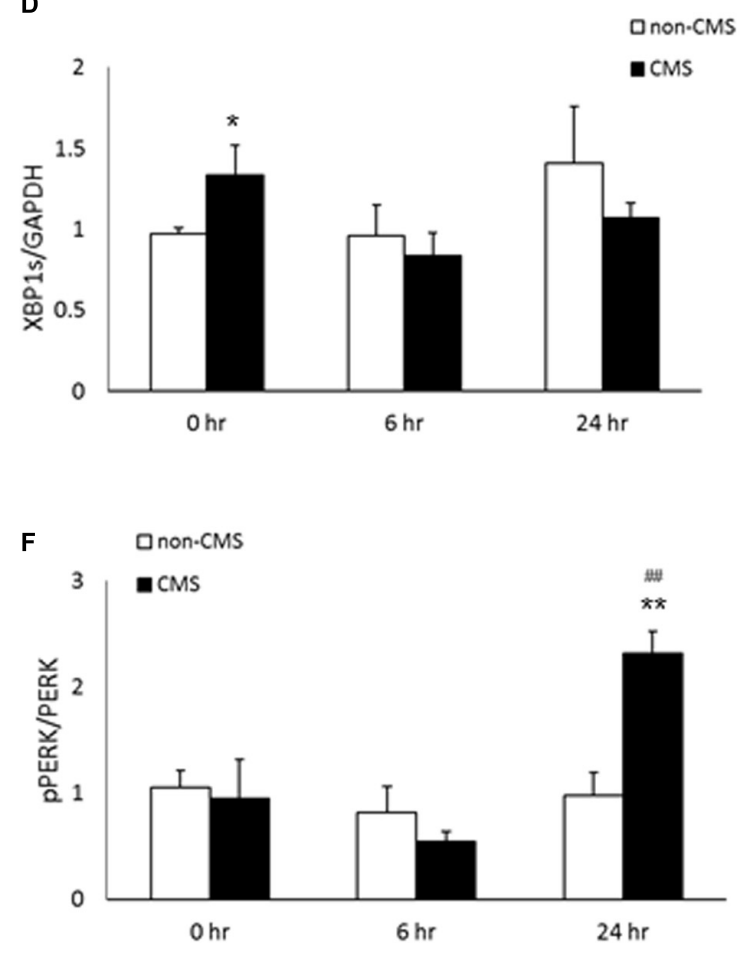

H

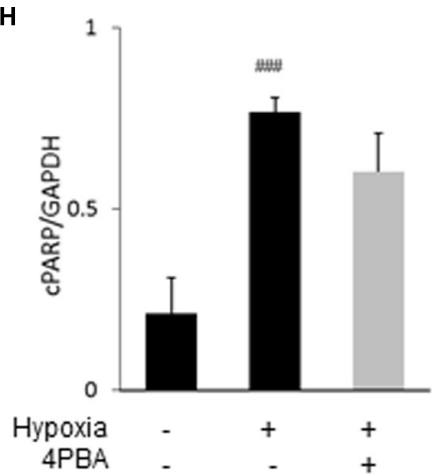

FIGURE 1 | ER stress in CMS neurons. (A) A representative blot of Grp78 in iPSC-derived non-CMS neurons $(n=3)$ and CMS neurons ( $n=3$ ) following hypoxia (1\% $\mathrm{O}_{2}$ ) treatment for 0,6 , and $24 \mathrm{~h}$. Tunicamycin (TM)-treated CMS neurons as a positive control for ER stress. (B) Densitometry analysis of Grp78. (C) A representative blot of XBP1s in iPSC-derived non-CMS neurons $(n=3)$ and CMS neurons $(n=3)$ following hypoxia $\left(1 \% \mathrm{O}_{2}\right)$ treatment for 0,6 , and 24 h. (D) Densitometry analysis of XBP1s. (E) A representative blot of PERK in iPSC-derived non-CMS neurons $(n=3)$ and CMS neurons $(n=3)$ following hypoxia (1\% $\left.\mathrm{O}_{2}\right)$ treatment for 0 , 6 , and 24 h. (F) Densitometry analysis of PERK. (G). A representative blot of PERK and cPARP in iPSC-derived CMS neurons following hypoxia (1\% $\mathrm{O}_{2}$ ) treatment for 0 and $48 \mathrm{~h}$ with or without $1 \mathrm{mM} 4$-PBA. (H) Densitometry analysis of cPARP. * indicates $p<0.05$ and ${ }^{* *}$ indicates $p<0.01$ as compared to non-CMS neurons at a given time point. ${ }^{\#}$ indicates $p<0.01$ and ${ }^{\# \# \#} p<0.001$ as compared to themselves under normoxia. 
A

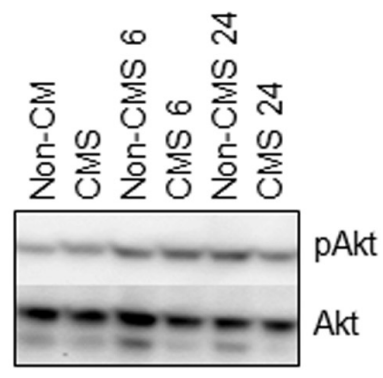

C

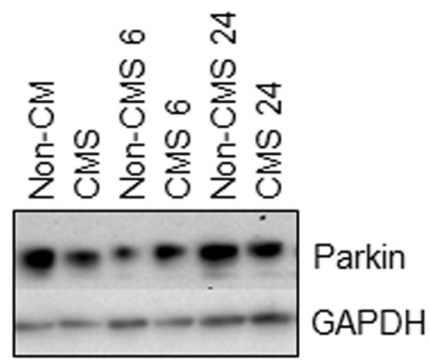

E

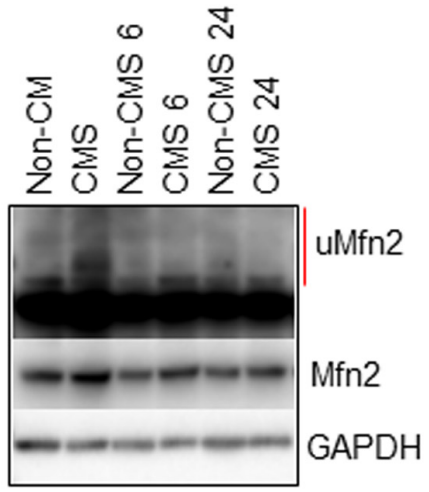

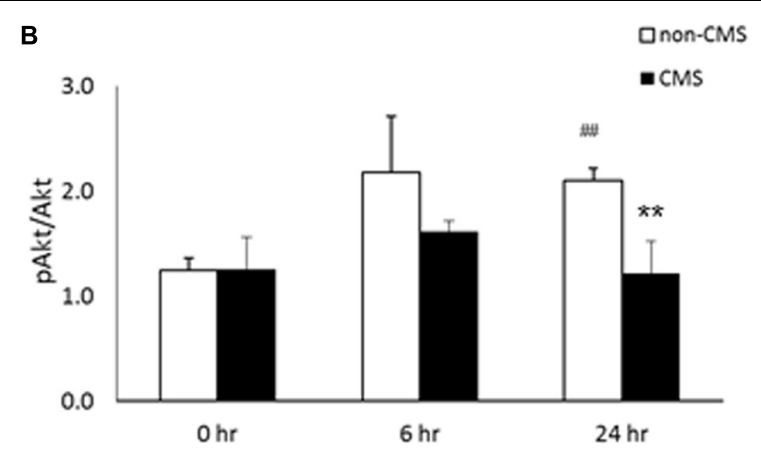

D anon-CMS

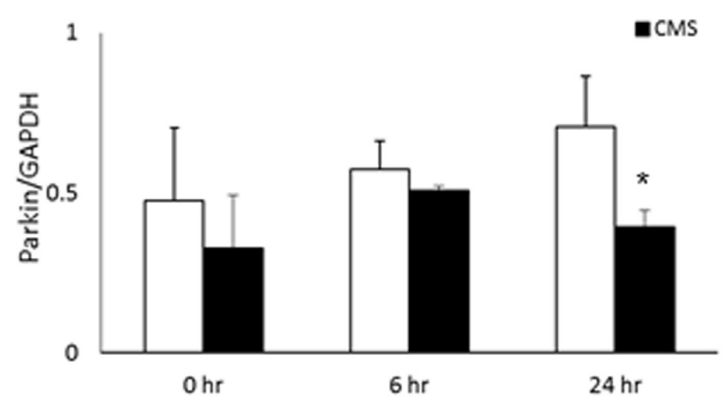

F

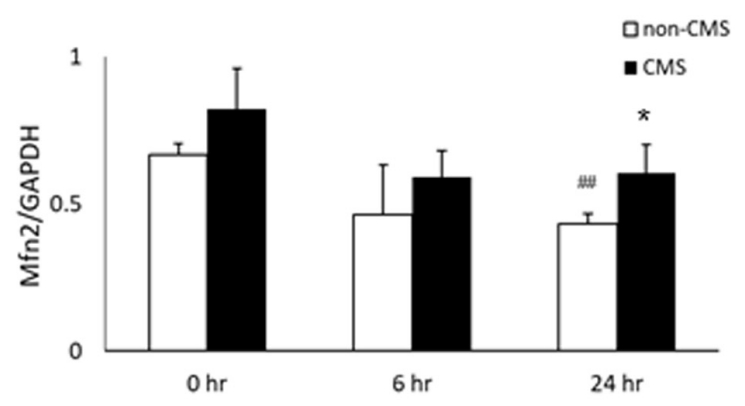

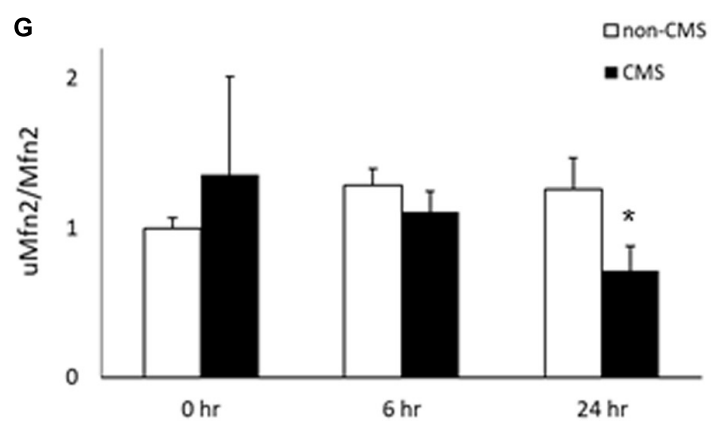

FIGURE 2 | Akt activation in CMS. (A) A representative blot of pAkt and Akt in iPSC-derived non-CMS neurons ( $n=3$ ) and CMS neurons $(n=3)$ following hypoxia $\left(1 \% \mathrm{O}_{2}\right)$ treatment for 0,6 , and $24 \mathrm{~h}$. (B) Densitometry analysis of Akt. (C) A representative blot of Parkin in iPSC-derived non-CMS neurons $(n=3)$ and CMS neurons $(n=3)$ following hypoxia $\left(1 \% \mathrm{O}_{2}\right)$ treatment for 0, 6, and 24 h. (D) Densitometry analysis of Parkin. (E) A representative blot of Mfn2 in iPSC-derived non-CMS neurons $(n=3)$ and CMS neurons $(n=3)$ following hypoxia ( $\left.1 \% \mathrm{O}_{2}\right)$ treatment for 0,6 , and $24 \mathrm{~h}$. Red line indicates the ubiquitylated Mfn2 (uMfn2). (F) Densitometry analysis of steady state of Mfn2 and (G) densitometry analysis of uMfn2. * indicates $p<0.05$ and ** indicates $p<0.01$ as compared to non-CMS neurons at a given time point. \#\# indicates $p<0.01$ as compared to themselves under normoxia. 
between non-CMS neurons and CMS neurons and this was significant at $24 \mathrm{~h}$ (Figures $2 \mathrm{C}, \mathbf{D},{ }^{*} p<0.05$ ), indicating that the changes of Parkin expression were not meditated through PINK1 signaling pathway.

\section{Hypoxia Decreases Mfn2 Expression in Non-CMS Neurons}

Mfn2 is a tether protein for ER-mitochondria contact and can be regulated by both Akt and Parkin. Overexpression of active Akt was shown to down-regulate Mfn2 (Dasgupta et al., 2015) and decreased Parkin expression was shown to increase steady state Mfn2 expression (Gautier et al., 2016; Lee et al., 2019). We compared the expression of Mfn2 and found that the baseline level of Mfn2 was similar between non-CMS and CMS neurons; however, hypoxia significantly decreased Mfn2 steady state expression in non-CMS (Figures $2 \mathbf{E}, \mathbf{F}, \# \# p<0.01$ ) but not in CMS neurons. Furthermore, the difference of Mfn2 expression between non-CMS neurons and CMS neurons was also significant at $24 \mathrm{~h}$ (Figures $2 \mathrm{E}, \mathbf{F},{ }^{*} p<0.05$ ). To determine whether a decreased Mfn2 steady state expression in nonCMS under hypoxia was due to an increased ubiquitinationmediated degradation, we compared the ratio of ubiquitylated Mfn2/non-ubiquitylated Mfn2 as described previously (Steffen et al., 2017; Mclelland et al., 2018). We found that non-CMS neurons have a significantly increased Mfn2 ubiquitination at $24 \mathrm{~h}$ (Figure 2G, ${ }^{*} p<0.05$ ), suggesting the presence of a PINK1independent ubiquitination.

\section{Enhanced Akt Activation and Parkin Overexpression Protect CMS Neurons From Hypoxia-Induced Cell Death}

Since both Akt activation and Parkin overexpression are protective mechanisms, we then examined whether lack of Akt activation or Parkin overexpression in CMS neurons leads to an increased susceptibility to hypoxia injury and cell death, we treated CMS neurons with (1) Akt agonist (insulin) and (2) an overexpression of Parkin with hypoxia for $48 \mathrm{~h}$. In these experiments, the expression level of cPARP was determined. Consistent with previous studies (Kim et al., 2011a; Zhao et al., 2017b), Akt activation was induced by insulin ( 2.1fold increase, $p<0.05$ ) and the Akt inhibitor MK2206 inhibits insulin-induced Akt activation (Figure 3A). Both increased Akt activation by insulin and overexpression of Parkin by lentivirus transduction ( $\sim 11$-fold increase, $p<0.01$, Figure 3B) significantly attenuated hypoxia-induced cell death in CMS neurons as compared to hypoxia exposure alone (Figures 3C,D, $\left.{ }^{*} p<0.05\right)$, confirming the protective role of Akt activation and Parkin overexpression in cell survival under hypoxia. The results are schematically summarized in Figure 3E.

\section{DISCUSSION}

In the current study, we report that hypoxia treatment induced an increased Akt activation and a decreased Mfn2 expression in non-CMS neurons. In contrast, hypoxia treatment results in an increased ER stress response in CMS neurons and lack of Akt activation and Parkin expression as compared to nonCMS neurons. Intriguingly, we found that by enhancing Akt activation and overexpressing Parkin in CMS neurons, hypoxiainduced CMS neuronal cell death was reduced, suggesting an important role of Akt signaling and Parkin overexpression in survival under hypoxia.

Stable and continuous oxygen supply to tissues is essential for the functional integrity of cells and survival of mammals, including humans. At high altitude, hypobaric hypoxia drives insufficient oxygen delivery to tissue and can result in hypoxic tissues. Hypoxia exposure results in many pathological consequences including cognitive impairment and contributes to a functional decline during aging (Yaffe et al., 2011; Babbar and Agarwal, 2012; Yeo, 2019). Interestingly, the majority of highlanders are able to adapt well and are considered as "healthy control." However, approximately $15-18 \%$ of highlanders cannot adapt to hypoxia and develop CMS, characterized by severe hypoxemia, excessive erythrocytosis and many neurologic manifestations including migraine, mental fatigue, confusion, and memory loss. Hence, CMS could be looked at as having manifestations of aging of high altitude in early adulthood (Sime et al., 1975).

Although the cellular mechanisms of CMS neuropathology remain largely unexplored, by using iPSC-derived neurons, we have previously demonstrated that CMS neurons have altered mitochondrial dynamics, fragmented mitochondria, a decreased ATP level and an increased susceptibility to hypoxiainduced cell death (Zhao et al., 2018). Of particular recent interest is that genomic analysis based on the same Andeans population from our laboratory has demonstrated that many ER stress-related genes are associated with hypoxia adaptation (Stobdan et al., 2017). Since the ER and mitochondria form a direct physical contact via mitochondria associated membrane (MAM), mitochondrial damage can lead to the activation of the ER stress response, and vice versa, ER stress can result in mitochondrial damage (Bouman et al., 2011; Senft and Ronai, 2015). Clinically, CMS patients experience exaggerated systemic oxidative-inflammatory-nitrosative stress (Bailey et al., 2019) and this is known to promote ER stress (Nakamura and Lipton, 2007; Eletto et al., 2014). Therefore, it is not surprising to find an increased expression of the ER stress marker Grp78 in CMS neurons even under normoxia. Under physiological conditions, Grp78 normally binds to ER proteins that constitute sensors for activation of three homeostatic pathways: IRE1, activating transcription factor-6 (ATF6) and PERK. The accumulation of unfolded proteins in the ER lumen during cellular stress conditions competes for Grp78 binding, and the dissociation of Grp78 from the sensor proteins initiates the ER stress signaling pathways aiming at removing the unfolded/misfolded proteins and alleviating stress to restore normal cellular function (Alder et al., 2005). Increased Grp78 is consistent with an accumulation of unfolded/misfolded proteins in the ER, and increased expression of XBP1s indicates that the IRE $1 \alpha$-mediated ER stress pathway was activated in CMS neurons. Previous studies have shown that overexpression of Grp78 may protect neurons and astrocytes from ischemic injury through activating either UPR/macroautophagy or Akt pro-survival pathway (Ouyang et al., 2011; Lu et al., 2015; Casas, 2017), but the 


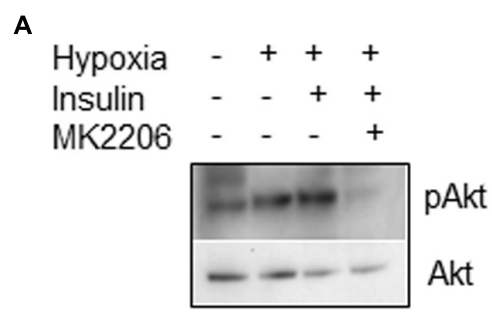

B

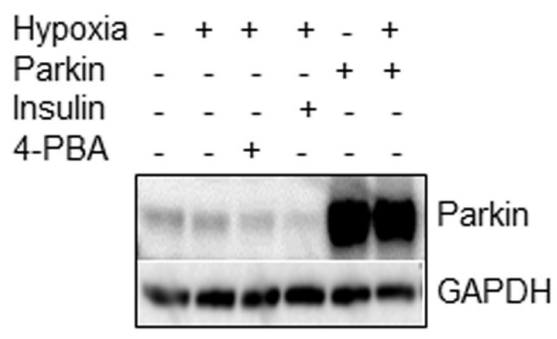

C

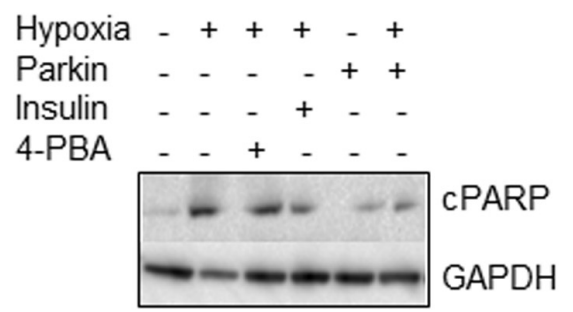

D

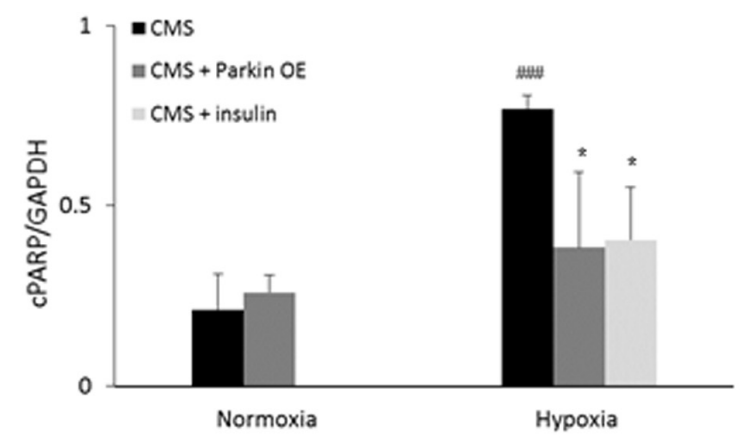

E

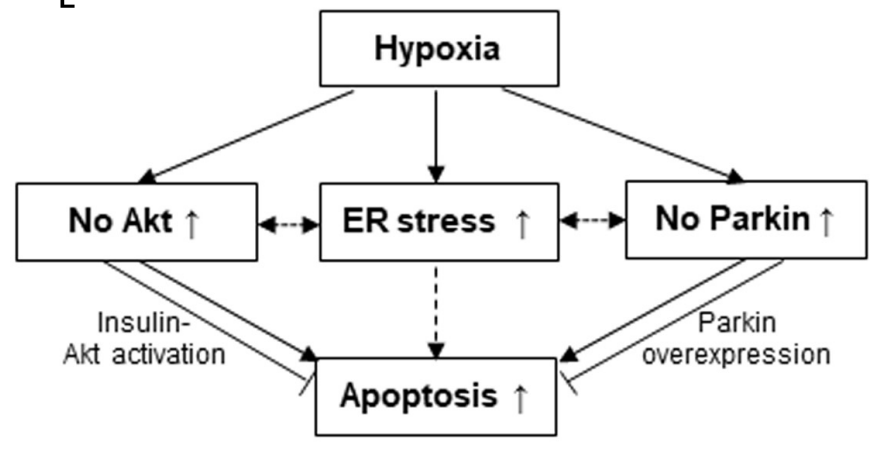

FIGURE 3 | Neuroprotective role of Akt and Parkin in CMS. (A) A representative blot of pAkt in iPSC-derived CMS neurons $(n=3)$ following hypoxia $\left(1 \% \mathrm{O}_{2}\right)$, hypoxia + $100 \mathrm{nM}$ insulin and hypoxia + $100 \mathrm{nM}$ insulin + MK2206 treatment for 48 h. (B) A representative blot of Parkin in iPSC-derived CMS neurons $(n=3)$ after a lentivirus transduction following hypoxia treatment or hypoxia with insulin or 4-PBA. (C) A representative blot of cPARP in iPSC-derived CMS neurons $(n=3)$ and Parkin-overexpressed CMS neuron $(n=3)$ following $1 \mathrm{mM} 4-\mathrm{PBA}, 100 \mathrm{nM}$ insulin and hypoxia $\left(1 \% \mathrm{O}_{2}\right)$ treatment for $48 \mathrm{~h}$. (D) densitometry analysis of cPARP (4-PBA data was summarized in Figure 1H). * indicates $p<0.05$ as compared to CMS neurons under hypoxia, \#\# $p<0.001$ as compared to themselves under normoxia. (E) Schematic overview of current findings. Hypoxic treatment results in an increased ER stress response and lack of Akt activation and Parkin expression in CMS neurons. Failure to activate Akt and expression of Parkin render CMS neurons more susceptible to hypoxia-induced cell death, which can be alleviated by insulin-induced Akt activation and Parkin overexpression. Solid line indicates findings obtained from the current study, and dashed lines indicate findings from literature.

prolonged UPR may lead to cell dysfunction and eventually cell death (Fernandez et al., 2015). Therefore, it is possible that overexpression of Grp78 might be one of the adaptive mechanisms in CMS neurons to protect themselves from an accumulated unfolded protein-induced toxicity. However, we cannot exclude that a higher baseline of Grp78 could indicate a higher steady state of ER stress in CMS neurons.

Hypoxia is known to induce ER stress through the accumulation of unfolded or misfolded proteins, both IRE1 $\alpha-$ XBP1 and PERK arm of the ER stress response are activates and toward repair or removal of the unfolded protein by either autophagy, ER associated degradation (ERAD) or apoptosis (Romero-Ramirez et al., 2004; Mujcic et al., 2013). In support, we found that the expression of Grp78, XBP1s, and phosphorylation of PERK trend to increase under hypoxia, but no difference of Grp78 and XBP1s was observed between non-CMS neurons and CMS neurons. The phosphorylation of PERK was more pronounced in CMS neurons compared to non-CMS neurons, suggesting that hypoxia in CMS neurons results in greater activation of the PERK pathway. It is well known that activation of the PERK pathway induces expression of CCAAT/-enhancerbinding protein homologus protein (CHOP) and other proapoptotic genes that can mediate cell death (Hiramatsu et al., 2015). However, interestingly enough, we were not able to detect CHOP expression in either CMS or non-CMS neurons under normoxia or hypoxia conditions. Although both tunicamycin and thapsigargin as ER inducers induced CHOP expression in CMS and non-CMS neurons (Supplementary Figure 2), these results indicate that, the increased hypoxia-induced cell death in CMS neurons was not mediated by CHOP. Furthermore, we found that treatment with 4-PBA, an ER stress alleviator, has a tendency to rescue hypoxia-induced cell death (Figures 1G,H) suggesting that ER stress alone may not play a major role in an increased cell death in CMS neurons.

Hypoxia induces an increase in Akt activation in nonCMS neurons but not in CMS neurons. Akt is a pro-survival signal and deactivation of Akt is a mediator of cell death (Luo et al., 2003). Akt activation is increased by short-term 
exposure to ER stress but is decreased by long-term exposure to ER stress (Hosoi et al., 2007). Therefore, we speculate that a higher baseline of ER stress (prolonged) prevents Akt activation in CMS neurons after hypoxia treatment and the lack of Akt activation in CMS results in an increased cell death. Our data further confirmed that enhancing Akt activation with insulin in CMS neurons can rescue hypoxia-induced cell death. PERK is a direct target of Akt and Akt phosphorylates PERK at threonine 799 and then inactivates PERK signaling (Hosoi et al., 2007; Mounir et al., 2011). Inhibition of Akt persistently activates the PERK pathway (Blaustein et al., 2013). Therefore, increased Akt activation in non-CMS neurons may prevent PERK activation and hence supports the idea that there was no ER stress in non-CMS neurons. Buroker and his colleagues found that Akt is one of the genes that are associated with chronic mountain sickness in Tibetans (Buroker et al., 2017). Our genome analysis from Andeans also identified SGK3 as one of top genes that are associated with CMS (Stobdan et al., 2017). SGK3 contains the same consensus substrate motif with Akt and shares common substrate targets, indicating that both Akt and SGK3 could substitute for each other or work synergistically (Castel and Scaltriti, 2017; Wang et al., 2019). Indeed, Wang et al. demonstrated that SGK3 silencing can induce ER stress and PERK overexpression in the aromatase inhibitor-resistant cancer cell line (Wang et al., 2017). Therefore, Akt and SGK3 might be other signaling proteins besides ER stress that can promote PERK activation in CMS neurons after hypoxia treatment. Interestingly, CMS bone marrow mononuclear cells were reported to have an increased Akt expression that promotes erythroblasts survival and decreases apoptosis in CMS patients with excessive erythrocytosis (Zhao et al., 2017a). The differences of Akt expression between neurons and bone marrow mononuclear cells in the two studies might be tissue-specific, since CMS patients have distinguishable tissue specific pathologies, namely in the central nervous system and in red blood cells (Azad et al., 2016; Zhao et al., 2018).

ER stress can induce Parkin expression and this expression protects cells from ER stress-induced cell death (Bouman et al., 2011). However, we did not observe an increased Parkin expression in CMS neurons as a possible result of increased ER stress under hypoxia, suggesting the potential loss of Parkin protection. In contrast, the level of Parkin expression in CMS neurons is significantly decreased as compared to that in the non-CMS neurons under hypoxia. Parkin loss of function increases the sensitivity of cells to ER stressinduced mitochondrial dysfunction and Parkin overexpression can protect cells from ER stress-induced mitochondrial damage through the maintenance of mitochondrial integrity (Bouman et al., 2011). Therefore, we believe that a decreased Parkin expression in CMS neurons under hypoxia render CMS neurons more sensitive to stress. Parkin overexpression increases retinal ganglion cell (RGC) survival in glaucomatous rats and partially restores the dysfunctional mitophagy (Dai et al., 2018). Parkin overexpression also attenuates dopaminergic neurodegeneration induced by 1-methyl-4-phenyl-1, 2, 3, 6-tetrahydropyridine (MPTP) through protecting the integrity of mitochondria (Bian et al., 2012). Indeed, our data confirmed that CMS neurons with Parkin overexpression attenuated the hypoxiainduced cell death, which might be due to the maintenance of mitochondrial integrity in CMS neurons under hypoxia. Parkin is also considered as a tumor suppressor. One of the tumor suppression mechanisms is mediated through regulating the hypoxia inducible factor (HIF) signaling pathway. Parkin interacts with HIF- $1 \alpha$ and promotes HIF- $1 \alpha$ degradation through ubiquitination. In many types of cancers, Parkin expression is frequently down regulated and therefore stabilizes HIF- $1 \alpha$ and promotes tumor metastasis and cancer development (Liu et al., 2017, 2018). In the current study, overexpression of Parkin in CMS neurons may promote HIF- $1 \alpha$ degradation and therefore lead to alleviation of hypoxia-induced injury as compared to those without overexpression of Parkin.

Mfn2 has been implicated in regulating mitochondrial dynamics, ER-mitochondrial tethering, mitophagy, mitochondrial metabolism and respiratory function, thus impacting cell fate and homeostasis (Bach et al., 2003; Eura et al., 2003; Schrepfer and Scorrano, 2016). Except for embryonic stem cell-induced neurons, degradation of $\mathrm{Mfn}$ is not required for mitophagy (Ordureau et al., 2020). In the current study, hypoxia induced a decreased expression of Mfn2 and increased Akt activation in non-CMS neurons. Akt activation has been shown to down-regulate Mfn2 (Dasgupta et al., 2015), therefore, a decreased Mfn2 expression in non-CMS neurons might be due to Akt activation. Hypoxia treatment has been shown to result in an increased Akt activation, a decreased Mfn2 expression and inhibition of mitochondrial apoptosis pathway in pulmonary artery smooth muscle cells (Fang et al., 2016). In another study, $\mathrm{H}_{2} \mathrm{O}_{2}$-induced oxidative stress led to concurrent increased Mfn-2 expression and apoptosis with profound inhibition of Akt activation in cultured neonatal rat cardiomyocytes (Shen et al., 2007). These observations suggested that Mfn2 and Akt are inversely correlated and activation of Akt and decreased Mfn2 may protect cells from oxidative stress-induced cell death, similar to what we observed in non-CMS neurons.

Mfn2 is a tether protein for ER and mitochondria and Parkin regulates the ER-mitochondria contact via Mfn2 (Basso et al., 2018). Although both Mfn 2 and Parkin have been shown to alter the ER-mitochondria coupling, it is still under debate whether the decreased Mfn2 and Parkin lead to a decrease or increase ER-mitochondrial coupling (De Brito and Scorrano, 2008; Schneeberger et al., 2013; Filadi et al., 2015; Gautier et al., 2016; Naon et al., 2016; Basso et al., 2018). The discrepancy between studies might be due to context and condition-specific factors. However, previous studies have demonstrated that decreased Mfn2 results in a reduced ER-mitochondria contact in neurons (Schneeberger et al., 2013; Gautier et al., 2016; Lee et al., 2018). For instance, Schneeberger et al. (2013) showed that ablation of Mfn2 in pro-opiomelanocortin neurons resulted in loss of ERmitochondria contacts, reduced oxygen consumption and energy expenditure. Gautier et al. (2016) demonstrated that (1) ERmitochondria contact and ER- to- mitochondrial $\mathrm{Ca}^{2+}$ transfer are enhanced in iPSC-derived neurons derived from patient with Parkin mutation, (2) Mfn2 expression is also increased in Parkin mutant neurons and Mfn2 down-regulation or the exogenous 
expression of Parkin can restore $\mathrm{Ca}^{2+}$ transients. In the current study, we observed a decrease in Mfn2 in the non-CMS neurons and we speculate that decreased Mfn2 may result in a decreased ER-mitochondrial contact and reduce energy expenditure, which might be one of adaptive mechanisms for non-CMS neurons surviving under hypoxia (Horscroft et al., 2017). It is worth noting that the decreased Mfn2 expression may also lead to mitochondrial fragmentation or increase ER stress (Filadi et al., 2018), therefore the precise role of Mfn 2 in non-CMS needs to be further investigated.

Altogether, we report that increased Akt activation acts as a protective or adaptive mechanism to prevent hypoxia-induced cell death in non-CMS neurons. In contrast, increased ER stress response may render CMS neurons more sensitive to stress; and the lack of protective mechanisms such as Akt activation and Parkin expression results in an increased cell death in CMS neurons under hypoxia. Our data provide evidence that loss of endogenous adaptive cytoprotection mechanisms such as impaired ER stress response and mitochondrial function contribute, at least in part, to the neuropathology in CMS patients.

\section{DATA AVAILABILITY STATEMENT}

Requests to access the datasets should be directed to $\mathrm{HZ}$, huzhao@ucsd.edu.

\section{ETHICS STATEMENT}

The studies involving human participants were reviewed and approved by under protocols approved by the University of

\section{REFERENCES}

Alder, N. N., Shen, Y., Brodsky, J. L., Hendershot, L. M., and Johnson, A. E. (2005). The molecular mechanisms underlying BiP-mediated gating of the Sec61 translocon of the endoplasmic reticulum. J. Cell. Biol. 168, 389-399. doi: $10.1083 /$ jcb. 200409174

Azad, P., Zhao, H. W., Cabrales, P. J., Ronen, R., Zhou, D., Poulsen, O., et al. (2016). Senp1 drives hypoxia-induced polycythemia via GATA1 and Bcl-xL in subjects with Monge's disease. J. Exp. Med. 213, 2729-2744. doi: 10.1084/jem.20151920

Babbar, R., and Agarwal, S. (2012). A new approach to hypobaric hypoxia induced cognitive impairment. Indian J. Med. Res. 136, 365-367.

Bach, D., Pich, S., Soriano, F. X., Vega, N., Baumgartner, B., Oriola, J., et al. (2003). Mitofusin-2 determines mitochondrial network architecture and mitochondrial metabolism. A novel regulatory mechanism altered in obesity. J. Biol. Chem. 278, 17190-17197. doi: 10.1074/jbc.m212754200

Bailey, D. M., Brugniaux, J. V., Filipponi, T., Marley, C. J., Stacey, B., Soria, R., et al. (2019). Exaggerated systemic oxidative-inflammatory-nitrosative stress in chronic mountain sickness is associated with cognitive decline and depression. J. Physiol. 597, 611-629. doi: 10.1113/jp276898

Bao, H., Wang, D., Zhao, X., Wu, Y., Yin, G., Meng, L., et al. (2017). Cerebral edema in chronic mountain sickness: a new finding. Sci. Rep. 7:43224.

Basso, V., Marchesan, E., Peggion, C., Chakraborty, J., Von Stockum, S., Giacomello, M., et al. (2018). Regulation of ER-mitochondria contacts by Parkin via Mfn2. Pharmacol. Res. 138, 43-56. doi: 10.1016/j.phrs.2018.09.006

Bian, M., Liu, J., Hong, X., Yu, M., Huang, Y., Sheng, Z., et al. (2012). Overexpression of parkin ameliorates dopaminergic neurodegeneration
California San Diego and the Universidad Peruana Cayetano Heredia. The patients/participants provided their written informed consent to participate in this study.

\section{AUTHOR CONTRIBUTIONS}

$\mathrm{HZ}$ and GH designed the studies. HZ carried out experiments and analyzed data. HZ, JL, GS, and GH wrote and edited the manuscript. All authors contributed to the article and approved the submitted version.

\section{FUNDING}

Our research is funded by the NIH grants R01 HL127403 and R01 HL146530 (to GH).

\section{SUPPLEMENTARY MATERIAL}

The Supplementary Material for this article can be found online at: https://www.frontiersin.org/articles/10.3389/fnins. 2020.607711/full\#supplementary-material

Supplementary Figure 1 | PINK1 and pS65-ubiquitin were not detected in non-CMS and CMS neurons. A representative blot of PINK1 and pS65-ubiquitin (redline) in iPSC-derived non-CMS neurons and CMS neurons following hypoxia $\left(1 \% \mathrm{O}_{2}\right)$ treatment for 0,6 , and $24 \mathrm{~h}, \mathrm{CCCP}$-treated PC3 cells as positive control.

Supplementary Figure 2 | CHOP was not detected in non-CMS and CMS neurons. A representative blot of $\mathrm{CHOP}$ in iPSC-derived non-CMS and CMS neurons following hypoxia $\left(1 \% \mathrm{O}_{2}\right)$ treatment for $0,24,48$, and $96 \mathrm{~h}$, thapsigargin (TG)-treated non-CMS neurons and tunicamycin (TM)-treated CMS neurons as positive control for ER stress and CHOP expression.

induced by 1- methyl-4-phenyl-1,2,3,6-tetrahydropyridine in mice. PLoS One 7:e39953. doi: 10.1371/journal.pone.0039953

Blaustein, M., Perez-Munizaga, D., Sanchez, M. A., Urrutia, C., Grande, A., Risso, G., et al. (2013). Modulation of the Akt pathway reveals a novel link with PERK/eIF2alpha, which is relevant during hypoxia. PLoS One 8:e69668. doi: 10.1371/journal.pone.0069668

Bouman, L., Schlierf, A., Lutz, A. K., Shan, J., Deinlein, A., Kast, J., et al. (2011). Parkin is transcriptionally regulated by ATF4: evidence for an interconnection between mitochondrial stress and ER stress. Cell Death Differ. 18, 769-782. doi: $10.1038 /$ cdd.2010.142

Buroker, N. E., Ning, X. H., Zhou, Z. N., Li, K., Cen, W. J., Wu, X. F., et al. (2017). SNPs, linkage disequilibrium, and chronic mountain sickness in Tibetan Chinese. Hypoxia (Auckl) 5, 67-74. doi: 10.2147/hp.s117967

Casas, C. (2017). GRP78 at the Centre of the Stage in Cancer and Neuroprotection. Front. Neurosci. 11:177.

Castel, P., and Scaltriti, M. (2017). The emerging role of serum/glucocorticoidregulated kinases in cancer. Cell Cycle 16, 5-6. doi: 10.1080/15384101.2016. 1232071

Dai, Y., Hu, X., and Sun, X. (2018). Overexpression of parkin protects retinal ganglion cells in experimental glaucoma. Cell Death Dis. 9:88.

Dasgupta, A., Chen, K. H., Munk, R. B., Sasaki, C. Y., Curtis, J., Longo, D. L., et al. (2015). Mechanism of activation-induced downregulation of Mitofusin 2 in human peripheral blood T cells. J. Immunol. 195, 5780-5786. doi: 10.4049/ jimmunol.1501023

De Brito, O. M., and Scorrano, L. (2008). Mitofusin 2 tethers endoplasmic reticulum to mitochondria. Nature 456, 605-610. doi: 10.1038/nature07534 
Delbrel, E., Soumare, A., Naguez, A., Label, R., Bernard, O., Bruhat, A., et al. (2018). HIF-1alpha triggers ER stress and CHOP-mediated apoptosis in alveolar epithelial cells, a key event in pulmonary fibrosis. Sci. Rep. 8:17939.

Eletto, D., Chevet, E., Argon, Y., and Appenzeller-Herzog, C. (2014). Redox controls UPR to control redox. J. Cell Sci. 127, 3649-3658. doi: 10.1242/jcs. 153643

Eura, Y., Ishihara, N., Yokota, S., and Mihara, K. (2003). Two mitofusin proteins, mammalian homologues of FZO, with distinct functions are both required for mitochondrial fusion. J. Biochem. 134, 333-344. doi: 10.1093/jb/m vg150

Fang, X., Chen, X., Zhong, G., Chen, Q., and Hu, C. (2016). Mitofusin 2 downregulation triggers pulmonary artery smooth muscle cell proliferation and apoptosis imbalance in rats with hypoxic pulmonary hypertension Via the PI3K/Akt and mitochondrial Apoptosis pathways. J. Cardiovasc. Pharmacol. 67, 164-174. doi: 10.1097/fjc.0000000000000333

Fernandez, A., Ordonez, R., Reiter, R. J., Gonzalez-Gallego, J., and Mauriz, J. L. (2015). Melatonin and endoplasmic reticulum stress: relation to autophagy and apoptosis. J. Pineal Res. 59, 292-307. doi: 10.1111/jpi.12264

Filadi, R., Greotti, E., Turacchio, G., Luini, A., Pozzan, T., and Pizzo, P. (2015). Mitofusin 2 ablation increases endoplasmic reticulum-mitochondria coupling. Proc. Natl. Acad. Sci. U.S.A. 112, E2174-E2181.

Filadi, R., Pendin, D., and Pizzo, P. (2018). Mitofusin 2: from functions to disease. Cell Death Dis. 9:330.

Gautier, C. A., Erpapazoglou, Z., Mouton-Liger, F., Muriel, M. P., Cormier, F., Bigou, S., et al. (2016). The endoplasmic reticulum-mitochondria interface is perturbed in PARK2 knockout mice and patients with PARK2 mutations. Hum. Mol. Genet. 25, 2972-2984.

Hiramatsu, N., Chiang, W. C., Kurt, T. D., Sigurdson, C. J., and Lin, J. H. (2015). Multiple mechanisms of unfolded protein response-induced cell death. Am. J. Pathol. 185, 1800-1808. doi: 10.1016/j.ajpath.2015.03.009

Horscroft, J. A., Kotwica, A. O., Laner, V., West, J. A., Hennis, P. J., Levett, D. Z. H., et al. (2017). Metabolic basis to Sherpa altitude adaptation. Proc. Natl. Acad. Sci. U.S.A. 114, 6382-6387.

Hosoi, T., Hyoda, K., Okuma, Y., Nomura, Y., and Ozawa, K. (2007). Akt up- and down-regulation in response to endoplasmic reticulum stress. Brain Res. 1152, 27-31. doi: 10.1016/j.brainres.2007.03.052

Iurlaro, R., Puschel, F., Leon-Annicchiarico, C. L., O'connor, H., Martin, S. J., Palou-Gramon, D., et al. (2017). Glucose deprivation induces ATF4-mediated Apoptosis through TRAIL death receptors. Mol. Cell Biol. 37, e479-e416.

Kabir, M. K., Kim, H. R., and Chae, H. J. (2018). Endoplasmic Reticulum Stress and Autophagy. London: Intech Open.

Kim, B., Mclean, L. L., Philip, S. S., and Feldman, E. L. (2011a). Hyperinsulinemia induces insulin resistance in dorsal root ganglion neurons. Endocrinology 152, 3638-3647. doi: 10.1210/en.2011-0029

Kim, J. E., O’sullivan, M. L., Sanchez, C. A., Hwang, M., Israel, M. A., Brennand, K., et al. (2011b). Investigating synapse formation and function using human pluripotent stem cell-derived neurons. Proc. Natl. Acad. Sci. U.S.A. 108, 30053010. doi: 10.1073/pnas.1007753108

Koh, D. W., Dawson, T. M., and Dawson, V. L. (2005). Mediation of cell death by poly(ADP-ribose) polymerase-1. Pharmacol. Res. 52, 5-14. doi: 10.1016/j.phrs. 2005.02.011

Lee, J., Choi, J. A., Cho, S. N., Son, S. H., and Song, C. H. (2019). Mitofusin 2-deficiency suppresses Mycobacterium tuberculosis survival in macrophages. Cells 8:1355. doi: 10.3390/cells8111355

Lee, K. S., Huh, S., Lee, S., Wu, Z., Kim, A. K., Kang, H. Y., et al. (2018). Altered ER-mitochondria contact impacts mitochondria calcium homeostasis and contributes to neurodegeneration in vivo in disease models. Proc. Natl. Acad. Sci. U.S.A. 115, E8844-E8853.

Li, K., Gesang, L., and He, C. (2017). Mechanism of apoptosis involved in gastric mucosal lesions in Tibetans with high-altitude polycythemia. Exp. Ther. Med. 14, 3780-3787. doi: 10.3892/etm.2017.4996

Lin, S. P., Li, W., Winters, A., Liu, R., and Yang, S. H. (2018). Artemisinin prevents glutamate-induced neuronal cell death Via Akt pathway activation. Front. Cell Neurosci. 12:108.

Liu, J., Zhang, C., Hu, W., and Feng, Z. (2018). Parkinson's disease-associated protein Parkin: an unusual player in cancer. Cancer Commun. (Lond) 38:40. doi: $10.1186 /$ s40880-018-0314-z
Liu, J., Zhang, C., Zhao, Y., Yue, X., Wu, H., Huang, S., et al. (2017). Parkin targets HIF-1alpha for ubiquitination and degradation to inhibit breast tumor progression. Nat. Commun. 8:1823.

Liu, L., Wu, H., Zang, J., Yang, G., Zhu, Y., Wu, Y., et al. (2016). 4phenylbutyric acid reveals good beneficial effects on vital organ function via anti-endoplasmic reticulum stress in septic rats. Crit. Care Med. 44, e689-e701.

Lu, T., Yang, W., Wang, Z., Hu, Z., Zeng, X., Yang, C., et al. (2015). Knockdown of glucose-regulated protein 78/binding immunoglobulin heavy chain protein expression by asymmetric small interfering RNA induces apoptosis in prostate cancer cells and attenuates migratory capability. Mol. Med. Rep. 11, 249-256. doi: $10.3892 / \mathrm{mmr} .2014 .2737$

Luo, H. R., Hattori, H., Hossain, M. A., Hester, L., Huang, Y., Lee-Kwon, W., et al. (2003). Akt as a mediator of cell death. Proc. Natl. Acad. Sci. U.S.A. 100, $11712-11717$.

Maekawa, H., and Inagi, R. (2017). Stress signal network between hypoxia and ER stress in chronic kidney disease. Front. Physiol. 8:74.

Mccoy, M. K., Kaganovich, A., Rudenko, I. N., Ding, J., and Cookson, M. R. (2014). Hexokinase activity is required for recruitment of parkin to depolarized mitochondria. Hum. Mol. Genet. 23, 145-156. doi: 10.1093/hmg/ddt407

Mclelland, G. L., Goiran, T., Yi, W., Dorval, G., Chen, C. X., Lauinger, N. D., et al. (2018). Mfn2 ubiquitination by PINK1/parkin gates the p97-dependent release of ER from mitochondria to drive mitophagy. eLife 7:e32866.

Mounir, Z., Krishnamoorthy, J. L., Wang, S., Papadopoulou, B., Campbell, S., Muller, W. J., et al. (2011). Akt determines cell fate through inhibition of the PERK-eIF2alpha phosphorylation pathway. Sci. Signal. 4:ra62. doi: 10.1126/ scisignal.2001630

Mujcic, H., Nagelkerke, A., Rouschop, K. M., Chung, S., Chaudary, N., Span, P. N., et al. (2013). Hypoxic activation of the PERK/eIF2alpha arm of the unfolded protein response promotes metastasis through induction of LAMP3. Clin. Cancer Res. 19, 6126-6137. doi: 10.1158/1078-0432.ccr-13-0526

Nakamura, T., and Lipton, S. A. (2007). Molecular mechanisms of nitrosative stress-mediated protein misfolding in neurodegenerative diseases. Cell Mol. Life Sci. 64, 1609-1620. doi: 10.1007/s00018-007-6525-0

Naon, D., Zaninello, M., Giacomello, M., Varanita, T., Grespi, F., Lakshminaranayan, S., et al. (2016). Critical reappraisal confirms that Mitofusin 2 is an endoplasmic reticulum-mitochondria tether. Proc. Natl. Acad. Sci. U.S.A. 113, 11249-11254. doi: 10.1073/pnas.1606786113

Ordureau, A., Paulo, J. A., Zhang, J., An, H., Swatek, K. N., Cannon, J. R., et al. (2020). Global landscape and dynamics of parkin and USP30-dependent ubiquitylomes in iNeurons during mitophagic signaling. Mol. Cell 77, 11241142.e1110.

Oslowski, C. M., and Urano, F. (2011). Measuring ER stress and the unfolded protein response using mammalian tissue culture system. Methods Enzymol. 490, 71-92. doi: 10.1016/b978-0-12-385114-7.00004-0

Ouyang, Y. B., Xu, L. J., Emery, J. F., Lee, A. S., and Giffard, R. G. (2011). Overexpressing GRP78 influences $\mathrm{Ca} 2+$ handling and function of mitochondria in astrocytes after ischemia-like stress. Mitochondrion 11, 279-286. doi: 10. 1016/j.mito.2010.10.007

Qin, L., Wang, Z., Tao, L., and Wang, Y. (2010). ER stress negatively regulates AKT/TSC/mTOR pathway to enhance autophagy. Autophagy 6, 239-247. doi: 10.4161/auto.6.2.11062

Romero-Ramirez, L., Cao, H., Nelson, D., Hammond, E., Lee, A. H., Yoshida, H., et al. (2004). XBP1 is essential for survival under hypoxic conditions and is required for tumor growth. Cancer Res. 64, 5943-5947. doi: 10.1158/0008-5472. can-04- 1606

Schneeberger, M., Dietrich, M. O., Sebastian, D., Imbernon, M., Castano, C., Garcia, A., et al. (2013). Mitofusin 2 in POMC neurons connects ER stress with leptin resistance and energy imbalance. Cell 155, 172-187. doi: 10.1016/j.cell. 2013.09.003

Schrepfer, E., and Scorrano, L. (2016). Mitofusins, from Mitochondria to Metabolism. Mol. Cell 61, 683-694. doi: 10.1016/j.molcel.2016.02.022

Senft, D., and Ronai, Z. A. (2015). UPR, autophagy, and mitochondria crosstalk underlies the ER stress response. Trends Biochem. Sci. 40, 141-148. doi: 10. 1016/j.tibs.2015.01.002

Shen, T., Zheng, M., Cao, C., Chen, C., Tang, J., Zhang, W., et al. (2007). Mitofusin-2 is a major determinant of oxidative stress-mediated heart muscle cell apoptosis. J. Biol. Chem. 282, 23354-23361. doi: 10.1074/jbc.m702657200 
Sime, F., Monge, C., and Whittembury, J. (1975). Age as a cause of chronic mountain sickness (Monge's disease). Int. J. Biometeorol. 19, 93-98. doi: 10. 1007/bf01463864

Soutar, M. P. M., Kempthorne, L., Miyakawa, S., Annuario, E., Melandri, D., Harley, J., et al. (2018). AKT signalling selectively regulates PINK1 mitophagy in SHSY5Y cells and human iPSC-derived neurons. Sci. Rep. 8:8855.

Steffen, J., Vashisht, A. A., Wan, J., Jen, J. C., Claypool, S. M., Wohlschlegel, J. A., et al. (2017). Rapid degradation of mutant SLC25A46 by the ubiquitinproteasome system results in MFN1/2-mediated hyperfusion of mitochondria. Mol. Biol. Cell 28, 600-612. doi: 10.1091/mbc.e16-07-0545

Stobdan, T., Akbari, A., Azad, P., Zhou, D., Poulsen, O., Appenzeller, O., et al. (2017). New Insights into the genetic basis of Monge's disease and adaptation to high-altitude. Mol. Biol. Evol. 34, 3154-3168. doi: 10.1093/molbev/msx239

Szegezdi, E., Logue, S. E., Gorman, A. M., and Samali, A. (2006). Mediators of endoplasmic reticulum stress-induced apoptosis. EMBO Rep. 7, 880-885. doi: 10.1038/sj.embor.7400779

Takahashi, K., Tanabe, K., Ohnuki, M., Narita, M., Ichisaka, T., Tomoda, K., et al. (2007). Induction of pluripotent stem cells from adult human fibroblasts by defined factors. Cell 131, 861-872. doi: 10.1016/j.cell.2007.11.019

Wang, H., Huang, F., Zhang, Z., Wang, P., Luo, Y., Li, H., et al. (2019). Feedback Activation of SGK3 and AKT contributes to rapamycin resistance by reactivating mTORC1/4EBP1 axis via TSC2 in breast cancer. Int. J. Biol. Sci. 15, 929-941. doi: 10.7150/ijbs.32489

Wang, Y., Zhou, D., Phung, S., Warden, C., Rashid, R., Chan, N., et al. (2017). SGK3 sustains ERalpha signaling and drives acquired aromatase inhibitor resistance through maintaining endoplasmic reticulum homeostasis. Proc. Natl. Acad. Sci. U.S.A. 114, E1500-E1508.

Wu, H., and Chen, Q. (2015). Hypoxia activation of mitophagy and its role in disease pathogenesis. Antioxid. Redox Signal. 22, 1032-1046. doi: 10.1089/ars. 2014.6204

Xu, L. H., Xie, H., Shi, Z. H., Du, L. D., Wing, Y. K., Li, A. M., et al. (2015). Critical role of endoplasmic reticulum stress in chronic intermittent hypoxia-induced deficits in synaptic plasticity and long-term memory. Antioxid. Redox Signal. 23, 695-710. doi: 10.1089/ars.2014.6122
Yaffe, K., Laffan, A. M., Harrison, S. L., Redline, S., Spira, A. P., Ensrud, K. E., et al. (2011). Sleep-disordered breathing, hypoxia, and risk of mild cognitive impairment and dementia in older women. JAMA 306, 613-619.

Yamashita, S. I., and Kanki, T. (2018). Detection of hypoxia-induced and iron depletion-induced mitophagy in mammalian cells. Methods Mol. Biol. 1759, 141-149. doi: 10.1007/7651_2017_19

Yeo, E. J. (2019). Hypoxia and aging. Exp. Mol. Med. 51:67.

Zhao, C., Li, Z., Ji, L., Ma, J., Ge, R. L., and Cui, S. (2017a). PI3K-Akt signal transduction molecules maybe involved in downregulation of erythroblasts apoptosis and perifosine increased its apoptosis in chronic mountain sickness. Med. Sci. Monit. 23, 5637-5649. doi: 10.12659/msm.905739

Zhao, H., Perkins, G., Yao, H., Callacondo, D., Appenzeller, O., Ellisman, M., et al. (2018). Mitochondrial dysfunction in iPSC-derived neurons of subjects with chronic mountain sickness. J. Appl. Physiol. (1985) 125, 832-840. doi: 10.1152/japplphysiol.00689.2017

Zhao, H. W., Gu, X. Q., Chailangkarn, T., Perkins, G., Callacondo, D., Appenzeller, O., et al. (2015). Altered iPSC-derived neurons' sodium channel properties in subjects with Monge's disease. Neuroscience 288, 187-199. doi: 10.1016/j. neuroscience.2014.12.039

Zhao, N., Liu, C. C., Van Ingelgom, A. J., Martens, Y. A., Linares, C., Knight, J. A., et al. (2017b). Apolipoprotein E4 impairs neuronal insulin signaling by trapping insulin receptor in the endosomes. Neuron 96, 115-129.e115.

Conflict of Interest: The authors declare that the research was conducted in the absence of any commercial or financial relationships that could be construed as a potential conflict of interest.

Copyright (c) 2021 Zhao, Lin, Sieck and Haddad. This is an open-access article distributed under the terms of the Creative Commons Attribution License (CC BY). The use, distribution or reproduction in other forums is permitted, provided the original author(s) and the copyright owner(s) are credited and that the original publication in this journal is cited, in accordance with accepted academic practice. No use, distribution or reproduction is permitted which does not comply with these terms. 